\title{
Transfer function analysis for clinical evaluation of dynamic cerebral autoregulation-a comparison between spontaneous and respiratory-induced oscillations
}

\author{
M Reinhard ${ }^{1}$, T Müller ${ }^{2}$, B Guschlbauer ${ }^{1}$, J Timmer $^{2}$ and A Hetzel ${ }^{1}$ \\ ${ }^{1}$ Department of Neurology and Clinical Neurophysiology, University of Freiburg, Germany \\ 2 Center for Data Analysis and Modeling, Department of Physics, University of Freiburg, \\ Germany
}

E-mail: HETZEL@nz11.ukl.uni-freiburg.de

\begin{abstract}
Oscillations of arterial blood pressure (ABP) and cerebral blood flow velocity $(\mathrm{CBFV})$ can be used for non-invasive assessment of cerebral autoregulation using transfer function analysis. Either spontaneous oscillations (SPO) around $0.1 \mathrm{~Hz}$ or respiratory induced oscillations during deep breathing (DB) at a rate of $6 / \mathrm{min}$ have been used so far. We investigated 168 patients with severe carotid stenosis or occlusion to evaluate transfer function analysis and compare the SPO and DB approaches. ABP was assessed non-invasively (Finapres), CBFV was measured in the middle cerebral artery using transcranial Doppler sonography. Transfer function phase $(P)$ and gain $(G)$ were extracted from the respective spectra in a low $(0.06-0.12 \mathrm{~Hz})$ and high $(0.20-0.30 \mathrm{~Hz})$ frequency range (LF, HF) of SPO and from the 0.1 (LF) and $0.2(\mathrm{HF}) \mathrm{Hz}$ peaks induced by DB. For SPO, significant side-to-side differences and differences between groups of severe and critical stenosis were found for $P(\mathrm{LF})$, while $P(\mathrm{HF}) \mathrm{did}$ not prove to be a significant parameter. $G(\mathrm{LF})$ showed significant side-to-side differences, while $G(\mathrm{HF})$ additionally differed significantly between severe and critical stenosis and occlusion, respectively. For DB, significant side-to-side differences were found for $P(\mathrm{LF}, \mathrm{HF})$. Mainly $G(\mathrm{HF})$ differed significantly between the affected and contralateral sides, while both HF and LF gains showed lower values in groups with a higher degree of stenosis. Correlation between $G$ and $P$ values was generally poor. Using Bland-Altman plots a poor inter-method agreement was found mainly for $P$. Correlations between SPO and DB were higher for $G$ than for $P$ (LF $r=0.64$ versus 0.44 , HF 0.69 versus 0.28 ). Analysing reproducibility in 16 patients, only for $P(\mathrm{LF}, \mathrm{HF})$ of $\mathrm{DB}$ was a highly significant correlation found (Spearman's $r$ up to 0.78 ). For $G(\mathrm{LF}, \mathrm{HF})$ correlations were significant for both SPO and DB with slightly higher $r$ coefficients for SPO. In conclusion, the present study showed that (1)
\end{abstract}


transfer functions $P$ and $G$ represent different information for characterization of dynamic cerebral autoregulation in the frequency domain. (2) Inter-method agreement between DB and SPO is poor for $P$ and moderate for $G$ values. (3) $P$ extracted from DB has a higher reproducibility. (4) The extraction of $P$ and $G$ from the SPO phase spectra is critical and future work on standardizing this process is needed. (5) At present, the DB protocol might be slightly advantageous as a routine diagnostic tool.

Keywords: cerebral autoregulation, carotid artery stenosis, $\mathrm{CO}_{2}$-reactivity, transcranial Doppler sonography, transfer function analysis

\section{Introduction}

Cerebral autoregulation guarantees blood supply of the brain relatively independent from changes in systemic arterial blood pressure (ABP). Graphically, this results in a plateau of the cerebral blood flow curve, the so-called autoregulatory plateau (Lassen 1959). Determination of the upper and lower limits of this plateau has been the method of choice for determination of autoregulatory capacity for many years. Since considerable manipulation of ABP is often needed, this approach could not be established as a clinical routine diagnostic tool (Panerai 1998). However, insight into cerebral autoregulatory capacity would be of interest in many clinical situations, both in terms of pathophysiological understanding and therapeutic management. Especially, detection of haemodynamic compromise in patients with severe carotid stenosis or acute ischaemic stroke might evolve as a major field of application.

It was not until the late 1980s, that our view on cerebral autoregulation changed substantially. Since then, insight into cerebral haemodynamics has become possible with a high temporal resolution by the advent of transcranial Doppler sonography (TCD). Using this method, analysis of both amplitude and time latencies of cerebral blood flow velocity (CBFV) in response to rapid changes in $\mathrm{ABP}$ has been performed (Aaslid et al 1989). This approach led to the understanding of cerebral autoregulation as 'dynamic cerebral autoregulation'. In a next step, besides provocation of rapid ABP changes by certain manoeuvres (e.g. rapid deflation of thigh cuffs or the Valsalva maneouvre) followed by analysis in the time domain, attention has been directed towards analysing physiologically occurring ABP changes, overruling the need of any external manipulation (Czosnyka et al 1996, Zhang et al 1998).

One of these non-invasive approaches analyses oscillations of ABP and CBFV in the frequency domain using transfer function analysis. The resultant phase and gain between the oscillations were suggested as a measure for dynamic cerebral autoregulatory capacity. Mainly a positive phase shift (i.e. CBFV oscillations precede ABP oscillations) in a low frequency (LF) range around $0.1 \mathrm{~Hz}$ and its reduction in a higher frequency (HF) range around 0.2$0.3 \mathrm{~Hz}$ was interpreted as intact cerebral autoregulation according to a high-pass filter model of the cerebral autoregulatory feedback control system (Diehl et al 1998). Furthermore, the transfer function gain between $\mathrm{ABP}$ and $\mathrm{CBFV}$ oscillations in the LF and HF ranges was found to be altered in pathological haemodynamic situations (Diehl et al 1998, Hu et al 1999). The LF oscillations around $0.1 \mathrm{~Hz}$ can either be evoked by deep breathing (DB) at a rate of 6/min (Diehl et al 1995), periodic squatting (Birch et al 1995) or by simply analysing spontaneous oscillations (SPO) around $0.1 \mathrm{~Hz}$ (so-called Mayer- or M-waves) during supine rest over several minutes (Hu et al 1999, Panerai et al 1998b, Zhang et al 1998). While the LF 
oscillations during DB are generated predominantly by respiratory-dependent periodic changes of intrathoracic pressure, SPO are most probably due to central oscillations in sympathetic nervous outflow (Myers et al 2001). The high frequency (HF) oscillations around $0.2-0.3 \mathrm{~Hz}$ (so-called respiratory- or R-waves) during SPO are due to the normal breathing rate (which lies between $12-18 / \mathrm{min}$ ) and are generated by the same respiratory-dependent mechanisms responsible for the LF oscillations during slow deep breathing at $6 / \mathrm{min}$, while $\mathrm{HF}$ oscillations at $0.2 \mathrm{~Hz}$ during DB are a higher harmonic of the primary DB oscillations.

Both SPO and DB have been used successfully for assessment of dynamic cerebral autoregulation using the transfer function analysis approach (Diehl et al 1998, Panerai et al 1998a). However, we are not aware that data from a large collective have been used to compare these methods.

\section{Methods}

\subsection{Patients and measurements}

Over a period of 2 years, 168 patients with severe uni- or bilateral internal carotid artery stenosis or occlusion were examined. The exclusion criteria included an insufficient temporal bone window (frequency in the studied patient population 15\%) and current atrial fibrillation (3\%). All patients underwent a complete neurosonological workup in our neurovascular lab, including extracranial and intracranial colour-coded and transcranial Doppler sonography. Grading of stenosis was performed using Doppler velocities pre-, intra- and poststenotically in combination with B-mode imaging (de Bray and Glatt 1995). Previous investigations in our department showed a high accuracy of ultrasonography $(\geqslant 93 \%)$ for determining high-grade carotid stenoses and occlusions (von Reutern and von Büdingen 1993, Hetzel et al 1998). Patients were assigned to different groups on the basis of the degree of stenosis (cf table 2). For analysis of reproducibility, 16 patients were reexamined after a mean interval of $4 \pm 4$ months. The degree of stenosis was confirmed to be unchanged beforehand.

Autoregulation measurements were performed with subjects in a supine position with $50^{\circ}$ inclination of the upper body. Cerebral blood flow velocity (CBFV) was measured in both middle cerebral arteries (MCA) by insonation through the temporal bone window with $2 \mathrm{MHz}$ transducers attached to a headband (DWL-Multidop- $\mathrm{X}^{\odot}$, Sipplingen, Germany). Continuous non-invasive ABP recording was achieved via a servo-controlled finger plethysmograph (Finapres ${ }^{\odot} 2300$, Ohmeda, Englewood, $\mathrm{CO}$ ) with the subject's right hand positioned at heart level. End-tidal $\mathrm{CO}_{2}$ partial pressure $\left(P_{\mathrm{ETCO}_{2}}\right)$ was measured in $\mathrm{mmHg}$ with an infrared capnometer $\left(\right.$ Normocap $^{\odot}$, Datex, Helsinki, Finland) during nasal expiration. $P_{\mathrm{ETCO}_{2} \text { values }}$ have been shown to correlate closely with intra-arterial $\mathrm{CO}_{2}$ values (Young et al 1991). After stable baseline values had been established, the servo mechanism of the Finapres device was turned off and a data segment of $10 \mathrm{~min}$ was recorded with the patients breathing spontaneously. After detailed instruction, sinusoidal oscillations in $\mathrm{ABP}$ and $\mathrm{CBFV}$ were elicited by breathing at a rate of $6 / \mathrm{min}$ (i.e. $5 \mathrm{~s}$ periods of in- and expiration) for $180 \mathrm{~s}$. In order to avoid hypocapnic conditions, persons were asked to breathe with rather small tidal volumes. A wrong rhythm (usually too short periods of inspiration) could normally be seen by the excursions of the thorax and the $P_{\mathrm{ETCO}_{2}}$ signal. The objective criterion for inability to perform DB was the failure of getting into a $6 / \mathrm{min}$ rhythm after a practicing period of $5 \mathrm{~min}$.

Haemodynamic parameters and $P_{\mathrm{ETCO}}$ levels were determined at the beginning and the end of the spontaneous and deep breathing periods. Finally, cerebrovascular reserve capacity was assessed using the $\mathrm{CO}_{2}$-reactivity test with inhalation of room air mixed with $7 \% \mathrm{CO}_{2}$. 
$\mathrm{CO}_{2}$-reactivity was calculated as the percentage increase of $\mathrm{CBFV}$ per $\mathrm{mmHg}$ increase of $P_{\mathrm{ETCO}_{2}}(\% / \mathrm{mmHg})$.

All parameters were recorded with a data-acquisition software package (TurboLab ${ }^{\odot}$ V4.3; Bresser Electronic, Munich, Germany) at a sampling rate of $100 \mathrm{~Hz}$ and further analysed using custom-written software developed in-house (T.M.).

\subsection{Data analysis}

2.2.1. Transfer function analysis. Mean length of analysed time series of ABP and CBFV was $554( \pm 84) \mathrm{s}$ for SPO and $179( \pm 20) \mathrm{s}$ for DB. From these given time series, the periodograms $P_{\mathrm{ABP}}(f)$ and $P_{\mathrm{CBFV}}(f)$ and the cross-periodogram $\mathrm{CP}(f)$ were computed by discrete Fourier transform of the whole data sets. For a dataset of length $T$, the resulting frequency resolution of the periodograms is $1 / T$. The auto-spectra $S_{\mathrm{ABP}}(f)$ and $S_{\mathrm{CBFV}}(f)$ and the cross-spectrum $\mathrm{CS}(f)$ were estimated by smoothing the respective periodograms with a triangular window of half-width eight frequency bins (Timmer et al 2000)

$$
\mathrm{CS}\left(f_{i}\right)=\sum_{j=-h}^{h} w_{j} \mathrm{CP}\left(f_{i+j}\right), \quad w_{j}=\frac{1}{h}-\frac{|j|}{h^{2}}, \quad h=8 .
$$

The resulting spectral estimate has a number $v$ of effective degrees of freedom given by

$$
v=2 \cdot\left(\sum_{i=-h}^{h} w_{i}^{2}\right)^{-1}
$$

(In our case of $h=8, v=23.8$. Note that we did not employ the Welch method of spectral estimation).

The phase spectrum $\varphi(f)$ is the argument of the (complex-valued) cross spectrum:

$$
\mathrm{CS}(f)=|\mathrm{CS}(f)| \exp (\mathrm{i} \varphi(f) .
$$

The coherence spectrum $\operatorname{Coh}(f)$ is defined as the normalized modulus of the cross spectrum:

$$
\operatorname{Coh}(f)=|\mathrm{CS}(f)| /\left(S_{\mathrm{ABP}}(f) S_{\mathrm{CBFV}}(f)\right)^{1 / 2} \text {. }
$$

Thus, the coherence at any frequency is a number between 0 and 1 , where 0 indicates no linear relationship and 1 indicates perfect linear dependence of the signals at the given frequency. The coherence is significantly different from 0 at significance level $\alpha(0.05$ in our case) if it is greater than the value

$$
s=\sqrt{ } 1-\alpha^{2 /(\nu-2)} \text {. }
$$

In our case, $s=0.49$ (Brockwell and Davis 1991).

The variance of the phase spectrum estimate $\varphi(f)$ is proportional to $\operatorname{Coh}(f)^{-2}$.

If the coherence is not significantly different from 0 , the phase spectrum cannot be used for analysis, since under the hypothesis of zero coherence, the phase spectrum is uniformly distributed over the interval $[-\pi, \pi]$ (Brockwell and Davis 1991). Therefore, a patient record was excluded from analysis if the peak value of coherence was not significantly different from zero.

For quantification of dynamical cerebral autoregulation the positive phase shift and the gain between ABP and CBFV was calculated.

For analysis of $\mathrm{DB}$, the dominant frequency peak at $0.1 \mathrm{~Hz}$ was selected in the LF range and the first multiple value at $0.2 \mathrm{~Hz}$ as the HF value for both sides. Determination of these frequencies was univocal.

The following rules were used for selection of phase and gain of SPO: (1) A point with maximum coherence (peak) within a core frequency range should be selected (core LF 
0.08-0.10 Hz, core HF 0.23-0.27 Hz; LF whole range 0.06-0.12 Hz, HF whole range 0.20$0.30 \mathrm{~Hz})$. (2) If several coherency peaks were present reaching nearly the same height $( \pm 0.1)$, the peak within or nearer to the core frequency range was selected. (3) Coherency peaks selected from the two MCA sides should be adjacent. (4) If there was one coherency peak on one MCA side and more than one on the other, then the peak nearest to the single peak on the one side was selected. (5) If a coherency plateau $>0.9$ was present reaching its maximum beyond the core frequency, then one bin of the plateau within or near the core frequency range was selected. (6) If a $>0.9$ coherency plateau was present within the core frequency range, frequencies of $0.09 \mathrm{~Hz}$ and $0.25 \mathrm{~Hz}$, respectively, were selected (usually only marginal changes of phase and gain were observed within such plateaus). (7) If a coherency peak was present reaching its maximum beyond the frequency range: in most cases, there was another still significant peak within the frequency range, which was then selected. If not, then a bin of significant coherency near the coherency peak on the other side was selected (usually of the ascending or descending slope of the coherency peak which reached its maximum beyond the frequency range). (8) If there was absolutely no coherency peak in the range on both sides but still significant coherency values (rare), then a bin of the ascending/descending slope of the coherency peak reaching its maximum beyond the frequency range was selected for both sides within the frequency range. (9) If phase wrap-around occurred at a coherency peak, that peak was not used for analysis and another coherency peak within the frequency range of interest was selected, if possible.

This procedure was applied blindly for the degree and side of stenosis.

2.2.2. Analysis of inter-method agreement and statistics. The phase and gain information from the two measurement protocols was analysed via Bland-Altman plots (Bland and Altman 1986). The Bland-Altman analysis consists of plotting the difference between two parameters (e.g. phase of DB and SPO) versus the mean of these two parameters. The distribution of the differences was checked for normality using $q-q$ plots. Given approximate normality, the $95 \%$ confidence interval for inter-method agreement is the mean \pm 1.96 standard deviations. A small mean indicates small inter-method bias, while a small variance indicates good intermethod agreement. Bland-Altman plots were also used to assess the reproducibility of both measurement protocols, plotting the two measured values against their mean. For assessment of reproducibility, Spearman's correlation coefficient was used additionally.

For analysis of intra- and interindividual differences Student's $t$-test and for correlating different parameters Pearson's coefficient were used. A $p$-value of less than 0.05 was considered statistically significant. Data are reported as mean $\pm \mathrm{SD}$.

\section{Results}

145 datasets of 129 patients ( $65 \pm 9$ yrs, 116 men) were included in the final analysis. 45 of the 190 initial datasets $(24 \%)$ had to be excluded due to lack of significant coherence during spontaneous oscillations in the low frequency band $(24 / 190,13 \%)$, inability to perform regular deep breathing $(17 / 190,9 \%)$ or artefacts of the Doppler or ABP signal during SPO $(4 / 190,2 \%)$.

\subsection{General results}

Values of ABP, HR and $P_{\mathrm{ETCO}_{2}}$ at baseline as well as changes during spontaneous oscillations (SPO) and deep breathing (DB) are given in table 1. An illustration of raw data and application of transfer function analysis is given in figures $1-3$. 
Table 1. Haemodynamic parameters and $P_{\mathrm{ETCO}_{2}}$ during SPO and DB.

\begin{tabular}{|c|c|c|c|c|c|}
\hline$n=145$ & & $\begin{array}{l}\text { Begin SPO } \\
\text { (=baseline) }\end{array}$ & $\begin{array}{l}\text { End SPO } \\
\text { (difference } \\
\text { to begin) }\end{array}$ & Begin DB & $\begin{array}{l}\text { End DB } \\
\text { (difference } \\
\text { from begin) }\end{array}$ \\
\hline $\mathrm{ABP}$ & $(\mathrm{mmHg})$ & $75.7 \pm 13.3$ & $-5.9 \pm 7.7^{b}$ & $71.4 \pm 13.1$ & $-0.7 \pm 6.4$ \\
\hline HR & (beats/min) & $68.5 \pm 11.6$ & $-0.1 \pm 2.9$ & $68.5 \pm 11.6$ & $3.2 \pm 6.5^{\mathrm{b}}$ \\
\hline $\begin{array}{l}\text { CBFV } \\
\text { ipsilateral }\end{array}$ & $\left(\mathrm{cm} \mathrm{s}^{-1}\right)$ & $45.2 \pm 10.7$ & $-0.5 \pm 2.5^{\mathrm{a}}$ & $42.4 \pm 9.7$ & $-0.8 \pm 3.8^{b}$ \\
\hline $\begin{array}{l}\text { CBFV } \\
\text { contralateral }\end{array}$ & $\left(\mathrm{cm} \mathrm{s}^{-1}\right)$ & $52.0 \pm 11.6$ & $-0.7 \pm 3.7^{\mathrm{a}}$ & $48.2 \pm 10.7$ & $-1.6 \pm 4.8^{b}$ \\
\hline$P_{\mathrm{ETCO}_{2}}$ & $(\mathrm{mmHg})$ & $38.0 \pm 4.0$ & $-0.1 \pm 2.8$ & $38.6 \pm 5.2$ & $-2.3 \pm 2.7^{b}$ \\
\hline
\end{tabular}

The results of the transfer function analysis of SPO, DB and $\mathrm{CO}_{2}$-reactivity in different groups of stenosis are shown in table 2. The mean analysed frequency in the LF and HF range of SPO did not differ significantly between different sides and groups. For SPO, significant side-to-side and inter-group differences were found for the LF phase, while the HF phase did not prove to be a significant parameter. LF gain showed significant side-to-side differences, while HF gain additionally differed significantly between severe and critical stenosis and occlusion, respectively. For DB, significant side-to-side differences were found for the LF and HF phase. Mainly HF gain differed significantly between the affected and contralateral sides, while both the HF and LF gains showed lower values in groups with a higher degree of stenosis. The correlation between the gain and phase values was not significant apart from $\operatorname{HF} \mathrm{DB}(r=0.15, P=0.01)$.

\subsection{Spontaneous oscillations (SPO) versus deep breathing (DB)}

In general, significantly higher phase shift values were observed for deep breathing (45.2 \pm $31.0^{\circ}$ versus $31.2 \pm 25.1^{\circ} ; p<0.001$, all datasets pooled, $n=290$ ). In comparison with SPO, $P_{\mathrm{ETCO}_{2}}$, and thus $\mathrm{CBFV}$, decreased highly significantly during $\mathrm{DB}$. Consequently, a significant negative correlation between the $P_{\mathrm{ETCO}_{2}}$ and LF phase was found for contralateral but not for affected sides during DB $(r=-0.36, P<0.001)$. Standard deviations were in the same range for both SPO and DB (cf table 2). The differentiation between different groups of stenosis using the LF phase did not show a clear superiority of one approach, while the HF phase was more useful using DB. For HF gain no major differences were found, while the LF gain showed better side-to-side but poorer inter-group differences for SPO.

Agreement of SPO and DB is illustrated in figure 4 by Bland-Altman plots. A considerable standard deviation for inter-method differences was observed for all parameters, indicating a poor inter-method agreement. If methods were compared using intra-individual side-to-side differences instead of the raw parameters, no better agreement was observed in Bland-Altman plots (not shown).

Correlations between phase and gain of SPO and $\mathrm{DB}$ as well as their correlation to $\mathrm{CO}_{2}$-reactivity are given in table 3 .

\subsection{Reproducibility}

Repeated measurements were performed in 16 patients (32 sides). The phase showed a more highly significant Spearman coefficient between the two measurements for DB, while gain correlated better for SPO (cf table 3). Bland-Altman plots showed a small 
(a) Spontaneous oscillations
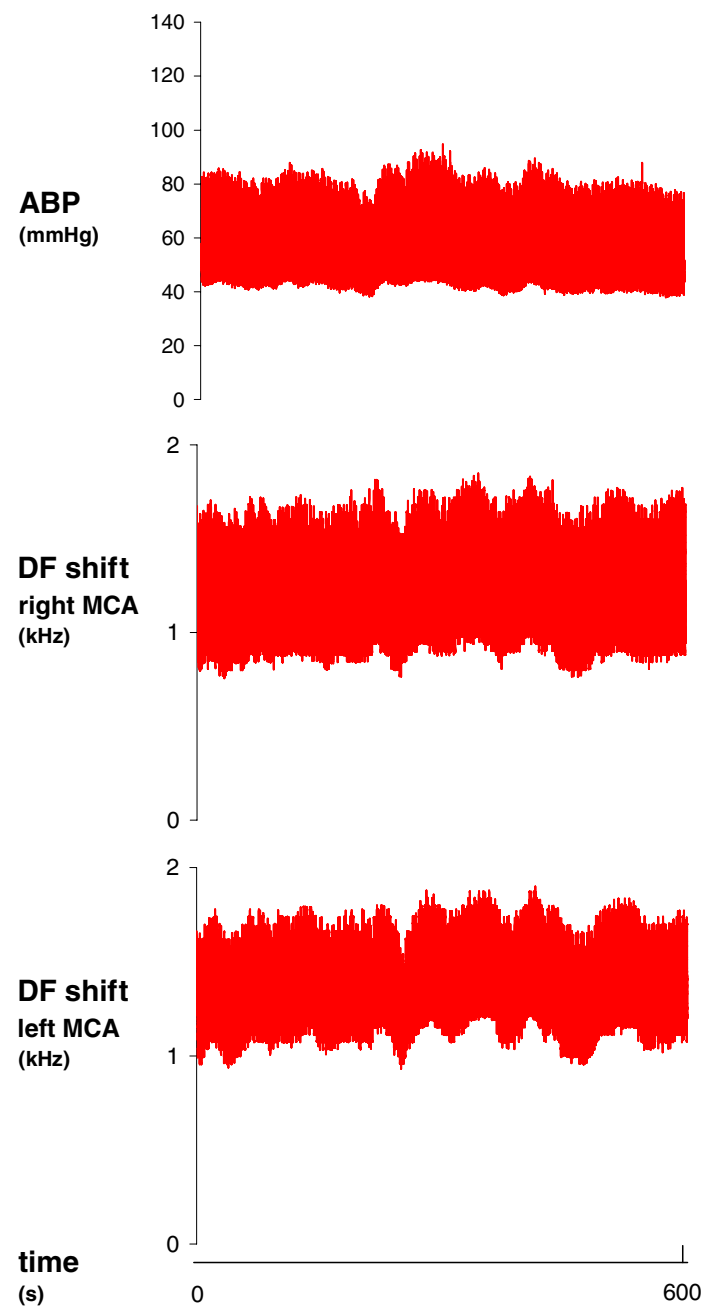

(b) Deep breathing
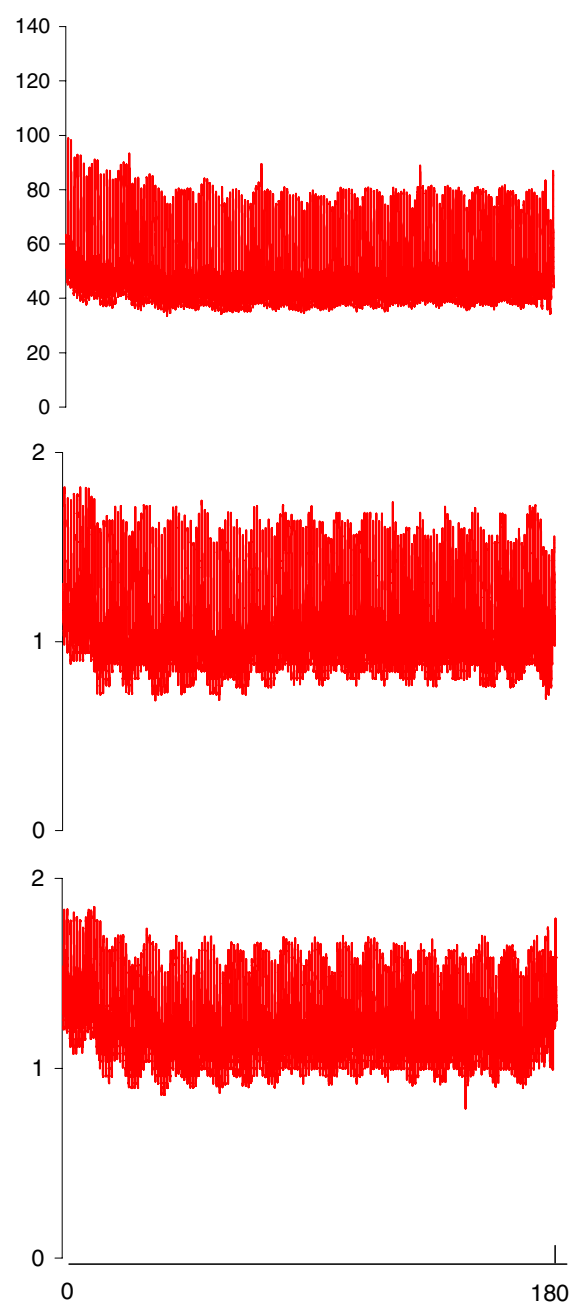

Figure 1. 64-yr-old patient with $80 \%$ stenosis of the left ICA. Illustrative recording of raw data (ABP in mmHg, Doppler frequency (DF) shift of the middle cerebral artery (MCA) in $\mathrm{kHz}$ ) during SPO (a) and DB (b). Note the clear sinusoidal oscillation during DB at a rate of $0.1 \mathrm{~Hz}$.

bias but a considerable variance for all transfer function parameters and $\mathrm{CO}_{2}$-reactivity (not shown).

\section{Discussion}

This study compares transfer function analysis of spontaneous oscillations (SPO) and deep breathing (DB) for the assessment of dynamic cerebral autoregulation. In addition, we present one of the largest collections of patients to date to whom the transfer function method has been 
power spectrum ABP $\left(\mathrm{mmHg}^{2} / \mathrm{Hz}\right)$
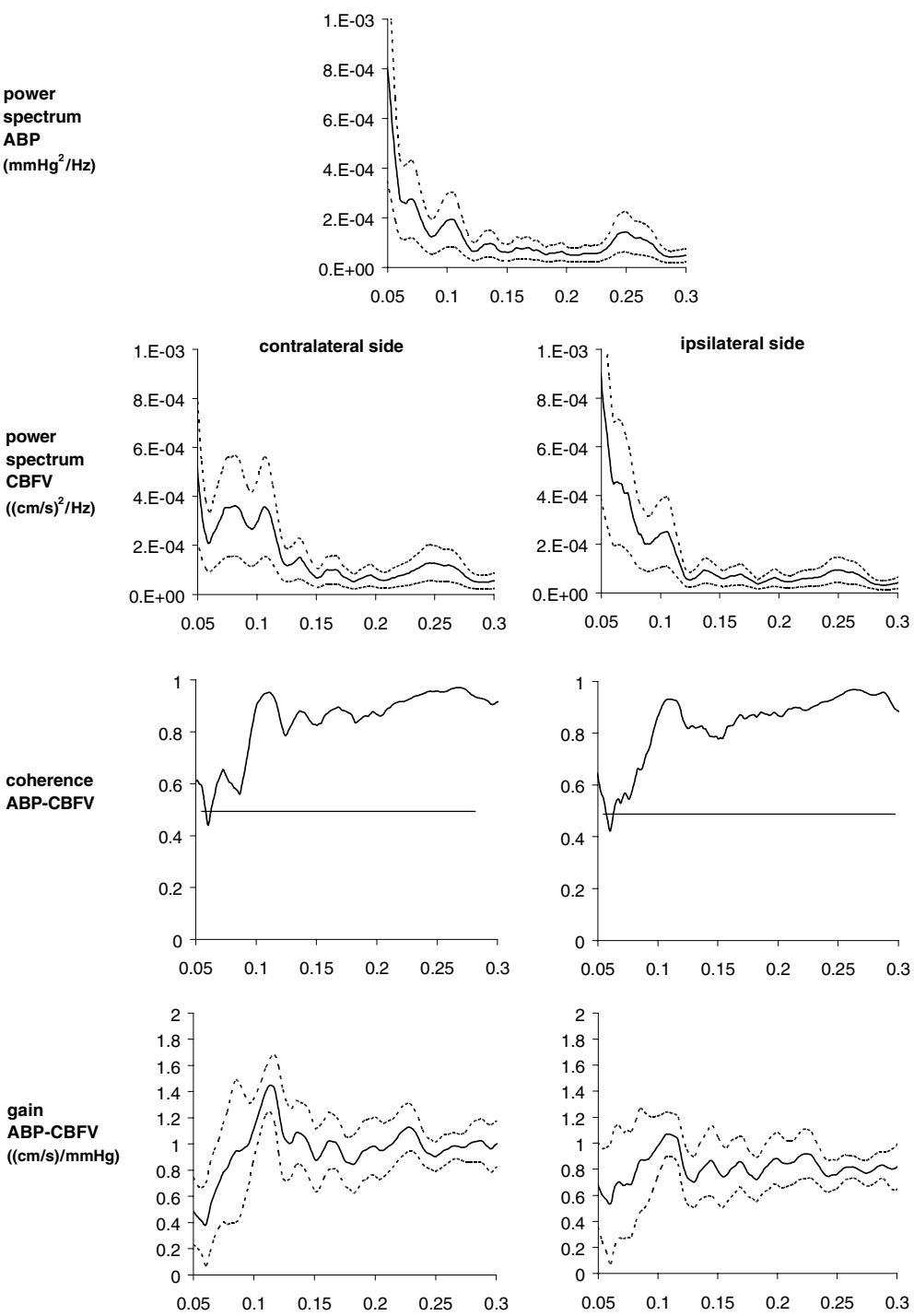
phase shift (degrees)

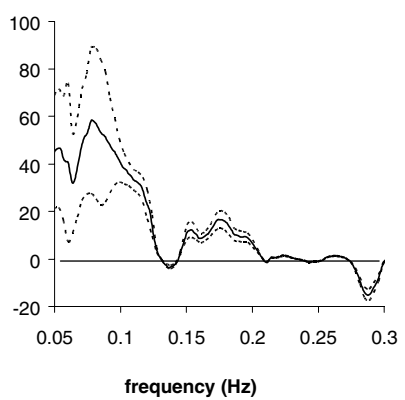

Figure 2. Transfer function analysis of the raw data (spontaneous oscillations) shown in figure 1. For the power spectra arbitrary units are given. Dotted lines represent the $95 \%$ confidence interval for the estimated parameters. For coherence spectra, the 0.05 level of significance $(0.49)$ is given by a horizontal line. In this case, phase and gain of SPO were selected at the bilateral coherence peak around $0.11 \mathrm{~Hz}$. 

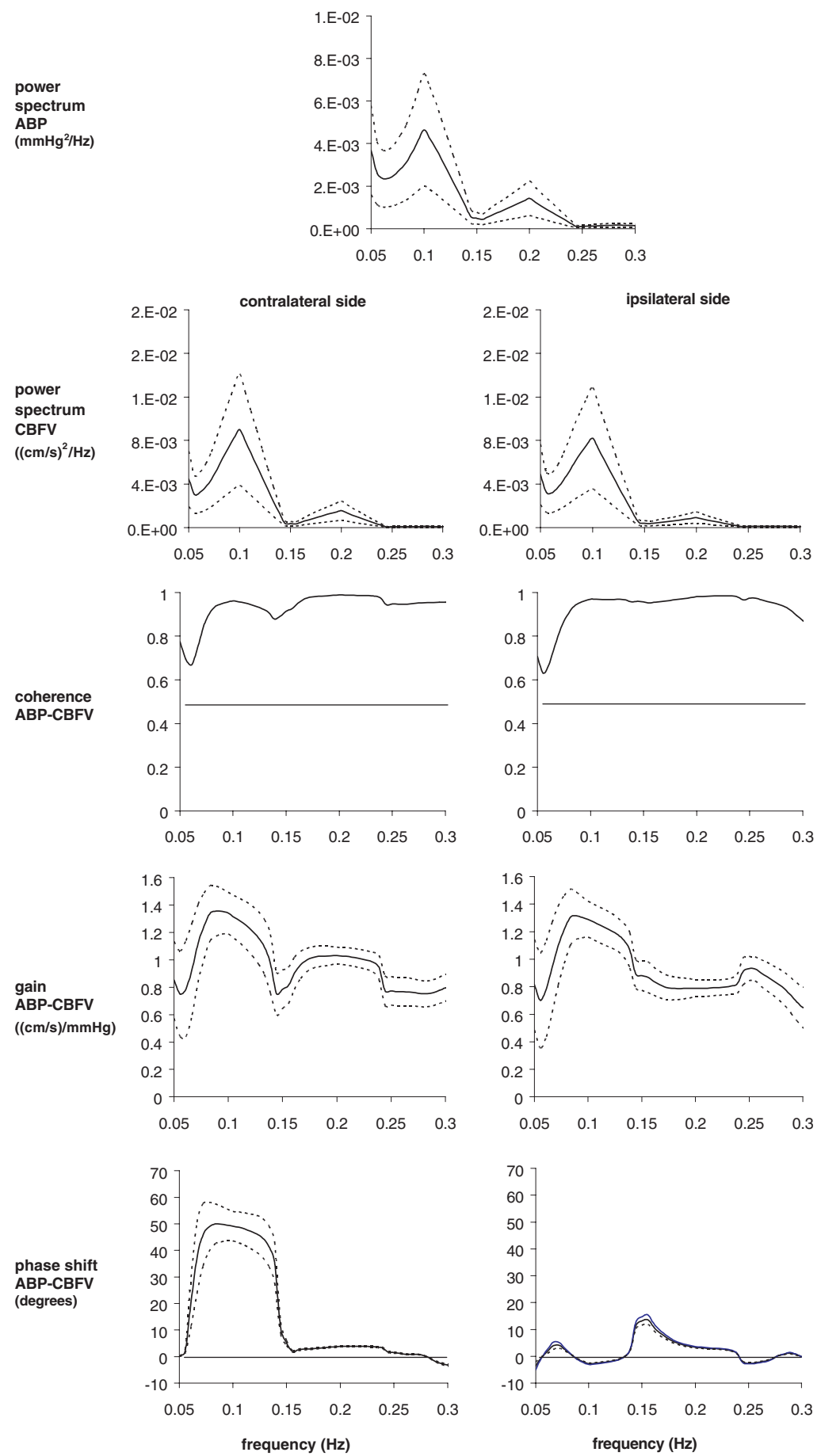

Figure 3. Transfer function analysis of the raw data (respiratory-induced oscillations: deep breathing) shown in figure 1. For the power spectra arbitrary units are given. Dotted lines represent the $95 \%$ confidence interval for the estimated parameters. For coherence spectra, the 0.05 level of significance $(0.49)$ is given by a horizontal line. In this case, phase and gain of SPO were selected at the bilateral coherence peak around $0.10 \mathrm{~Hz}$. 
Table 2. Results of transfer function analysis and $\mathrm{CO}_{2}$-reactivity. A-D: sub-groups with different degrees of internal carotid artery stenosis. A: unilateral stenosis of $75-89 \%$, B: $90-99 \%$, C: $100 \%$, D: bilateral stenosis of $>80 \%$ (the side of higher stenosis was defined as the 'ipsilateral' side). For SPO, the mean analysed low frequency was $0.092 \pm 0.017 \mathrm{~Hz}$, while the mean high frequency was $0.256 \pm 0.020 \mathrm{~Hz}$ (no significant differences between both sides). For DB, the analysed frequencies $(0.1$ and $0.2 \mathrm{~Hz}$, respectively) were given by the breathing rate of $0.1 \mathrm{~Hz}$.

$$
n=129
$$

Spontaneous oscillation Low frequency range Phase shift (deg)

Ipsilateral

Contralateral

Gain $\left(\left(\mathrm{cm} \mathrm{s}^{-1}\right) / \mathrm{mmHg}\right)$

Ipsilateral

Contralateral

High frequency range

Phase shift (deg)

Ipsilateral

Contralateral

Gain $\left(\left(\mathrm{cm} \mathrm{s}^{-1}\right) / \mathrm{mmHg}\right)$

Ipsilateral

Contralateral

\section{Deep breathing}

Low frequency

range $(0.1 \mathrm{~Hz})$

Phase shift (deg)

Ipsilateral

Contralateral

Gain $\left(\left(\mathrm{cm} \mathrm{s}^{-1}\right) / \mathrm{mmHg}\right)$

Ipsilateral

Contralateral

High frequency

range $(0.2 \mathrm{~Hz})$

Phase shift (deg)

Ipsilateral

Contralateral

Gain $\left(\left(\mathrm{cm} \mathrm{s}^{-1}\right) / \mathrm{mmHg}\right)$

Ipsilateral

Contralateral

$\mathrm{CO}_{2}$-reactivity $(\% \mathrm{mmHg})$

Ipsilateral

Contralateral

$$
\text { A }(n=44)
$$$$
\text { 75-89\% }
$$

B $(n=43)$
$90-99 \%$

$\mathrm{C}(n=24)$

$100 \%$

$\mathrm{D}(n=18)$

Bilateral

stenosis $>80 \% \quad$ Significances
$35.0 \pm 24.0$

$44.0 \pm 21.7$

$19.6 \pm 19.9$

$38.6 \pm 31.2$

$24.3 \pm 24.5$

$41.5 \pm 22.5$

$14.4 \pm 17.8$

$23.1 \pm 23.3$

A-B: $p=0.002 ; \mathrm{A}-\mathrm{D}: p=0.002$

A-D: $p=0.001$; B-D: $p=0.038$;

C-D: $p=0.014$

Ipsi versus contra

A: $p=0.005 ; \mathrm{B}: p<0.001 ; \mathrm{C}: p=0.001$

$0.57 \pm 0.23$

$0.74 \pm 0.36$

$0.48 \pm 0.23$

$0.69 \pm 0.36$

$0.42 \pm 0.19$

$0.53 \pm 0.22$

$0.45 \pm 0.47$

$0.46 \pm 0.40$

NS

NS

Ipsi versus contra

A, B: $p<0.001 ;$ C: $p=0.006$

$10.7 \pm 20.9$

$15.0 \pm 17.6$

$11.4 \pm 15.2$

$12.9 \pm 21.1$

$6.8 \pm 21.0$

$13.2 \pm 19.1$

NS

NS

Ipsi versus contra: A: $p=0.02$

$0.73 \pm 0.24$

$1.02 \pm 0.36$

$0.58 \pm 0.21$

$1.07 \pm 0.34$

$0.51 \pm 0.27$

$1.00 \pm 0.36$

$0.53 \pm 0.47$

$0.67 \pm 0.58$

A-B: $p=0.021 ; \mathrm{A}-\mathrm{C}: p=0.005$

NS

Ipsi versus contra: A, B, C: $p<0.001$
$45.8 \pm 28.7$

$55.0 \pm 28.6$

$31.6 \pm 26.2$
$65.6 \pm 33.1$

$31.2 \pm 31.6$

$48.2 \pm 25.5$

$33.3 \pm 29.1$

$39.2 \pm 33.9$

$0.50 \pm 0.18$

$0.68 \pm 0.21$

$0.76 \pm 0.34$

$0.74 \pm 0.37$

$18.2 \pm 16.3$

$26.0 \pm 21.0$

$6.3 \pm 16.9$

$23.9 \pm 16.2$

$12.2 \pm 10.9$

$21.7 \pm 19.0$

$11.2 \pm 11.4$

$25.2 \pm 12.2$

$0.71 \pm 0.31$

$0.97 \pm 0.47$

$0.56 \pm 0.27$

$0.50 \pm 0.24$

$0.86 \pm 0.30$

$0.49 \pm 0.41$

$0.65 \pm 0.52$

$1.73 \pm 0.75$

$2.15 \pm 0.66$
$1.06 \pm 0.70$

$1.97 \pm 0.66$
$1.36 \pm 1.00$

$2.30 \pm 0.94$
$1.01 \pm 0.79$

$1.60 \pm 0.82$
A-B: $p=0.018$

NS

Ipsi versus contra A: $p=0.001$;

B: $p<0.001 ; \mathrm{C}: p=0.025$

A-C: $p=0.002 ;$ B-C: $p=0.025$

NS

Ipsi versus contra C: $p<0.001$

A-B: $p=0.001$

NS

Ipsi versus contra A: $p=0.002$

B, D: $p<0.001$; C: $p=0.035$

A-B: $p=0.021 ; \mathrm{A}-\mathrm{C}: p=0.005$

NS

Ipsi versus contra: A, B, C: $p<0.001$

A-B: $p<0.001 ; \mathrm{A}-\mathrm{D}: p=0.001$

A-D: $p=0.017$

Ipsi versus contra: A, B, C: $p<0.001$;

D: $p=0.006$ 
Table 3. Results of correlation analysis.

$\begin{array}{lcclcc} & & & \text { Coefficient } & \text { Significance } \\ \text { SPO/DB with } \mathrm{CO}_{2} \text {-reactivity }(n=290 \text { sides }) & & & \text { Pearson } & \\ \text { Phase } & \text { SPO } & \text { LF } & 0.32 & <0.001 \\ & & \text { HF } & 0.06 & \text { NS } \\ & \text { DB } & \text { LF } & 0.38 & <0.001 \\ & & \text { HF } & 0.39 & <0.001 \\ \text { Gain } & \text { SPO } & \text { LF } & 0.16 & 0.005 \\ & & \text { HF } & 0.26 & <0.001 \\ & \text { DB } & \text { LF } & 0.09 & \text { NS } \\ & & \text { HF } & 0.33 & <0.001 \\ \text { SPO versus DB }(n=290 \text { sides }) & & & & \\ \text { Phase } & & \text { LF } & 0.44 & <0.001 \\ & & \text { HF } & 0.28 & <0.001 \\ \text { Gain } & & \text { LF } & 0.64 & <0.001 \\ & & \text { HF } & 0.69 & <0.001 \\ \text { Reproducibility }(n=32 \text { sides }) & & & \text { Spearman } & \\ \text { Phase } & \text { SPO } & \text { LF } & 0.38 & 0.031 \\ & & \text { HF } & 0.03 & \text { NS } \\ & \text { DB } & \text { LF } & 0.78 & <0.001 \\ \text { Gain } & & \text { HF } & 0.63 & <0.001 \\ & \text { SPO } & \text { LF } & 0.68 & <0.001 \\ & & \text { HF } & 0.68 & <0.001 \\ & \text { DB } & \text { LF } & 0.52 & 0.002 \\ & & \text { HF } & 0.60 & <0.001 \\ & & & & \\ & & & \end{array}$

applied. Both the SPO and the DB approach have been used in a number of studies for grading dynamic cerebral autoregulatory capacity based on a high-pass filter model of the cerebral autoregulatory feedback system (Panerai 1998). Especially the transfer function phase has been reported to represent cerebral autoregulatory capacity (Diehl et al 1998). In addition, the magnitude or gain of the transfer function has also been examined, but showed less clear differentiation between normal and pathologic states (Panerai et al 1998a). Other authors reported in a recent model-based study on the additional usefulness of transfer function gain in assessing autoregulatory capacity (Kirkham et al 2001).

\subsection{Practical aspects of deep breathing $(D B)$ and spontaneous oscillations (SPO)}

Analysing spontaneous oscillations seems to be the simplest and least cumbersome method for assessment of cerebral autoregulation in a clinical setting. Its practical application in acute or chronically ill patients, however, might be hampered by the fact that not all patients are capable of lying still for a period of ten minutes without causing measurement artefacts both of the Doppler and Finapres signals. Especially for the Finapres device, a deterioration of the blood pressure signal after more than $7 \mathrm{~min}$ without allowing the device to servo-control itself is not rare (see ABP in table 1). In addition, in patients with frequent extrabeats or atrial fibrillation, detection of SPO is difficult and all previous studies have been done excluding these patients. Finally, spontaneous oscillations in the low frequency range (M-waves) appear inconsistently and even have a relatively high intraindividual day-to-day variability 
(a) LF phase

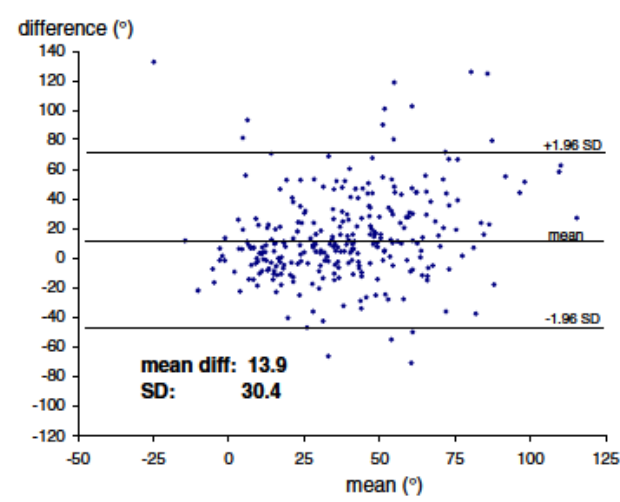

(c) LF gain

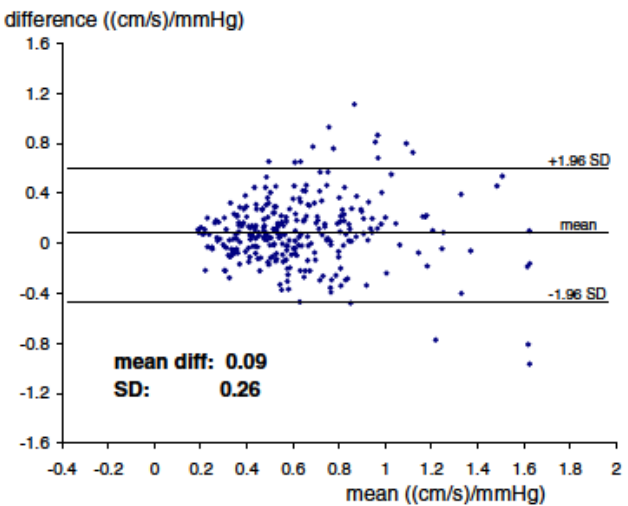

(b) HF phase

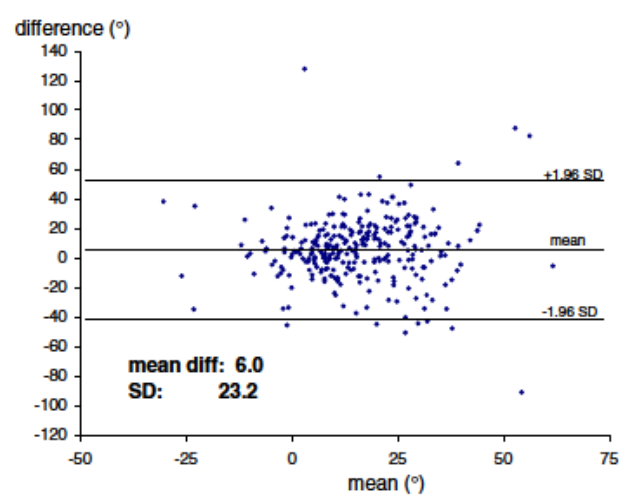

(d) HF gain

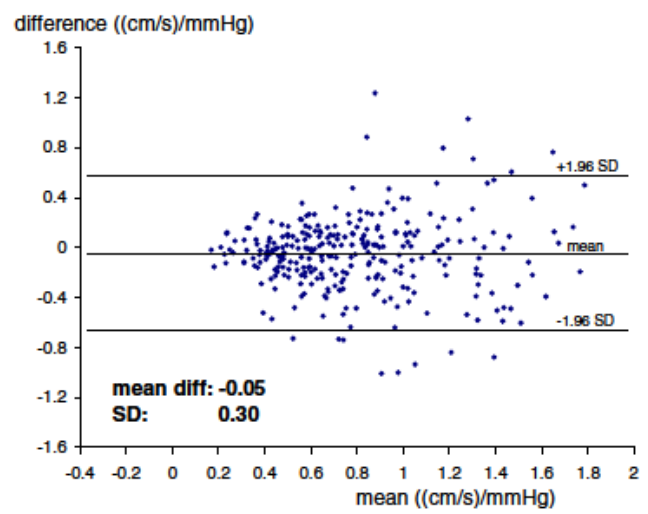

Figure 4. Bland-Altman plots. Comparison between spontaneous oscillations and deep breathing $(n=290)$. The mean of the two measurements is plotted against the difference. The mean difference (DB minus SPO), its standard deviation (SD) and the 95\% confidence interval (i.e. 1.96 SD) are given in each figure.

(Dimier-David et al 1994). Besides a low signal-to-noise ratio, this lack of significant M-waves might be the main reason for the lack of coherence in $13 \%$ of all SPO recordings.

The DB method is less time consuming and guarantees the same frequency to be analysed in all patients. Significant coherence was observed in all patients. The limiting factor for clinical application of this method was the inability of the patients in this collective to breathe regularly at a rate of $6 / \mathrm{min}$. This was mainly due to a kind of 'respiratory apraxia', less often communication problems were present (aphasia, foreign language) or the limited respiratory rate with a larger tidal volume was not tolerated due to underlying pulmonary disease. Interestingly, however, this method can even be applied to sedated and ventilated patients by using a 6 per minute ventilation protocol (Lang et al 2001). A disadvantage of the DB approach is obviously the fact that patients tend to become hypocapnic due to the larger tidal volume during deep breathing. $P_{\mathrm{ETCO}_{2}}$ has been shown to influence both static and dynamic cerebral autoregulation measurements (Panerai et al 1999). In the present study a significant correlation between phase values of the contralateral sides and $P_{\mathrm{ETCO}}$ values was found. This correlation was not found for the affected side (reduced reactivity to $\mathrm{CO}_{2}$ ) and for 
SPO. The original application of 'deep' or 'forced' breathing was indeed to elicit large tidal volumes with large intrathoracic pressure changes and thus marked R-waves for autonomic function testing (heart rate variability). For use as a cerebral autoregulation test, this might be prevented by strict instruction to breathe slowly with small tidal volumes, but not all patients are able to do so.

\subsection{Methodological aspects and limitations of transfer function analysis}

For routine clinical application a diagnostic tool has to be observer-independent and easy to handle. Analysis of the phase spectrum is univocal for the DB approach, since there is nearly always a clear peak at the target frequency of $0.1 \mathrm{~Hz}$ (cf figure 1(b)). However, looking at figure 2, evaluation of the phase spectrum calculated from SPO is more difficult and selection of a single frequency for extraction of the phase and gain information may be problematic both in terms of physiological validity and of inter-observer agreement. Thus, the method of phase and gain extraction is one of the key points in applying the SPO approach and might have significantly influenced the results of the present study. Previous investigators suggested the point of maximum coherence within the interesting frequency range to be analysed (Hu et al 1999). The maximum coherency guarantees a high reliability of the calculated phase and gain parameters. On the other hand, efficient autoregulation might exactly be reflected by a low coherency (Giller 1990). This is obviously a dilemma of the SPO approach. One might even speculate that a total lack of significant coherency within the analysed frequency range (as observed in $13 \%$ of our patients) might be a sign of preserved autoregulation. Interestingly, the problem of missing coherency was more pronounced on the affected side. Cerebrovascular reactivity in the patients excluded due to missing coherency, however, was $1.20 \pm 0.87 \% / \mathrm{mmHg}$ on the affected side. This does not support the hypothesis of excellent autoregulation in this group of patients.

For extraction of the phase and gain information from the SPO spectrum, we mostly adhered to the rule of $\mathrm{Hu}$ and colleagues and selected the gain and phase information at the point of maximum coherency (see the proposed criteria in section 2). Two main additional points are relevant in our criteria: first, the problem of using different frequencies for different patients (e.g. 0.06 versus $0.12 \mathrm{~Hz}$ ) was avoided by giving priority to a 'core frequency' range. Second, we considered the problem of different coherency maxima of the two sides (which could affect intraindividual side-to-side differences). Establishing our criteria, we tried to avoid undue subjective interference, and a relatively constant frequency selection was possible. However, this issue still remains a problem and part of the results might have been influenced by the difficulties in interpreting the spectral results. In the future, the development of a generally accepted algorithm such as averaging of a frequency range (weighed by different factors) rather than picking a single frequency might merit further efforts.

The HF phase and gain extraction during SPO was less difficult, since there was usually a broad coherency peak around $0.25 \mathrm{~Hz}$, representing the range of the respiratory frequency. During DB, a HF peak naturally occurs at $0.2 \mathrm{~Hz}$ as the first multiple value. This frequency is obviously lower than the HF range of SPO, which could have influenced the results. However, the difference is quite systematic and in our opinion it does not represent a major flaw but should well be kept in mind when interpreting the results.

Phase wrap-around (i.e. a jump of the phase by $360^{\circ}$ ) was rarely observed in the frequency ranges of interest (i.e. in five of the 190 recordings). If it occurred, selection of another coherency peak was usually possible (see criterion (8) in section 2). This phenomenon was therefore not the main factor influencing the phase and gain extraction from the respective spectra in the present study and no special phase unwrapping algorithm was needed. Phase 
wrap-around is more of an issue for a future automated procedure of phase extraction, while during manual analysis phase wrap-arounds can easily be identified in given plots of the respective phase spectra.

\subsection{Interpretation of general results}

Applying transfer function analysis to different groups of carotid stenosis, significant differences between the contralateral and affected sides were found for both DB and SPO. Transfer function phase was more powerful in detecting group and side-to-side differences in the LF range, while transfer function gain seemed to be more useful in the HF range. Standard deviations were in the same relative range for gain and phase values. Though both phase and gain showed significant pathological values on the affected side in patients with severe carotid stenosis, their correlation was very poor. Therefore, transfer function gain and phase might contain different information for characterizing dynamic cerebral autoregulation in the frequency domain. Correlation with $\mathrm{CO}_{2}$-reactivity, which represents a relatively well-established parameter of cerebral haemodynamic compromise, revealed significant correlations for HF gain and LF (DB also HF) phase. However, the $r$ coefficients were only poor to moderate and the HF phase of SPO and LF gain of DB showed no significant correlations. Dynamic cerebral autoregulation characterized in the frequency domain is thus not adequately reflected by a standard $\mathrm{CO}_{2}$-reactivity test. The different tests seem to assess different aspects of haemodynamic compromise.

In the largest study to date $(n=83)$ applying the SPO transfer function analysis to patients with carotid artery stenosis, Hu and colleagues (1999) found transfer function phase to be severely reduced below $10^{\circ}$ (with a high SD of up to $38^{\circ}$ ) both in $90-99 \%$ stenosis and carotid occlusion. Correlations with $\mathrm{CO}_{2}$-reactivity were moderate in this study.

The DB method has not been applied to a comparable number of patients up to now. Our phase results are in line with values of our previous study in patients with carotid artery disease (Reinhard et al 2001), but they are still lower than the values originally observed by Diehl and colleagues for carotid obstructions (Diehl et al 1995). However, in the latter study a different patient profile including more younger patients with therapeutic occlusion of the ICA than patients with atherosclerotic carotid artery stenosis was examined (Diehl et al 1998).

Transfer function gain is in general thought to reflect the relation of amplitude changes between $\mathrm{ABP}$ oscillations as input function and CBFV oscillations as output function. As already found in the study of Hu et al (1999), transfer function gain in the high frequency range was more useful in marking off different sides and groups of stenosis. HF gain seems to be more sensitive by indicating lacking high-pass filter properties. In contrast to the contralateral sides which show increasing gain with higher frequencies, the HF gain on the affected sides remains the same. Interestingly, absolute values of transfer function gain both in the HF and LF range were significantly smaller on the affected side, as observed by previous investigators (Hu et al 1999, Diehl et al 1991, Bazner et al 1995). At first sight, a small gain could be due to intact dampening effects of amplitude changes in ABP. With pressure passive perfusion as expected with dilated cerebral arterioles downstream a severe carotid stenosis, one would rather assume an increased gain compared with the unaffected side. However, it is comprehensible that the inability to achieve active diameter changes in (sub-)maximally dilated arterioles leads to reduced CBFV amplitude oscillations (Diehl et al 1991). Probably, spontaneous oscillations of cerebral perfusion pressure do not reach amplitude ranges comparable to those generated by additional active autoregulatory oscillations of arteriolar diameter on the unaffected side. 


\subsection{Comparing results of deep breathing $(D B)$ and spontaneous oscillations (SPO)}

More parameters calculated from DB than from SPO showed significant results in differentiating between groups of stenosis and different sides (cf table 2). For standard deviations no relevant differences were found and correlations with $\mathrm{CO}_{2}$-reactivity did not reveal a clear superiority of one method.

We compared the SPO and DB approach by the widely-used Bland-Altman plots (cf figure 3). A central finding is that inter-method agreement for the single individual in terms of variance of the differences was generally moderate to poor. Another point is that gain obviously showed a better correlation between DB and SPO than phase.

The inter-method bias with higher LF phase (and gain) on DB might be predominantly explained by the accompanying hypocapnia during DB (see above). Explanation of the poor phase agreement is more difficult. One point might be that during deep breathing mainly mechanical causes are responsible for the generation of $0.1 \mathrm{~Hz}$ oscillations in ABP, whereas spontaneous oscillations at $0.1 \mathrm{~Hz}$ are based on complex feedback mechanisms of the autonomic nervous system ('sympathetic gain') (Preiss and Polosa 1974). Therefore, interference with both inter- and intraindividually varying autonomic modulation might be thinkable. Another, more methodological point is the often difficult interpretation of the SPO phase spectrum compared with the very consistent peak at $0.1 \mathrm{~Hz}$ generated by DB. Despite our efforts to standardize interpretation of phase spectra (see section 4.3), this might still have considerably hampered the SPO results. It is remarkable that transfer function gain showed a better inter-method correlation both for LF and HF (cf table 3). Thus, transfer function gain seems to be in general more robust when comparing SPO and DB, even in the light of slightly different HF ranges.

When comparing the clinical applicability of two methods, analysis of reproducibility also is of interest. Analysing the two different measurements, only for DB (LF and HF range) was a highly significant intra-individual correlation for phase values found. Analysing the gain, correlations were significant for both approaches and frequency ranges with slightly higher $r$ coefficients for SPO. These results underline the view that phase shift extracted from DB is more stable than SPO phase and that transfer function gain might be more reliably obtained from SPO recordings.

\subsection{Conclusions}

The present study showed that: (1) grading of dynamic cerebral autoregulation is possible by transfer function analysis using both SPO and DB. (2) Transfer function gain and phase represent different information for characterization of dynamic cerebral autoregulation in the frequency domain. (3) Inter-method agreement between DB and SPO is poor for phase and moderate for gain values. (4) Phase extracted from DB has a higher reproducibility, and given the unsolved methodological problems in interpreting SPO phase spectra as well as practical aspects, it might be slightly advantageous as a routine diagnostic tool. (5) For a possible broader clinical use of SPO, analysis of gain should be routinely included due to its higher robustness. In addition, further work on standardizing the phase extraction procedure for SPO has to be done. Finally, other mathematical approaches to spontaneous and respiratory-induced oscillations of cerebral haemodynamics and autoregulation not restricted to the frequency domain will have to be evaluated regarding inter-method agreement and reproducibility. 


\section{Acknowledgment}

MR and TM acknowledge support from the German Federal Ministry of Education and Research $(b m b+f)$.

\section{References}

Aaslid R, Lindegaard K F, Sorteberg W and Nornes H 1989 Cerebral autoregulation dynamics in humans Stroke 20 45-52

Birch A A, Dirnhuber M J, Hartley-Davies R, Iannotti F and Neil-Dwyer G 1995 Assessment of autoregulation by means of periodic changes in blood pressure Stroke 26 834-7

Bland J M and Altman D G 1986 Statistical methods for assessing agreement between two methods of clinical measurement Lancet 1 307-10

Brockwell P J and Davis R A 1991 Time Series: Theory and Methods (New York: Springer)

Czosnyka M, Smielewski P, Kirkpatrick P, Menon D K and Pickard J D 1996 Monitoring of cerebral autoregulation in head-injured patients Stroke 27 1829-34

de Bray J M and Glatt B 1995 Quantification of atheromatous stenosis in the extracranial internal carotid artery Cerebrovasc. Dis. 5 414-26

Derdeyn C P, Videen T O, Fritsch S M, Carpenter D A, Grubb R L Jr and Powers W J 1999 Compensatory mechanisms for chronic cerebral hypoperfusion in patients with carotid occlusion Stroke 30 1019-24

Diehl R R, Diehl B, Sitzer M and Hennerici M 1991 Spontaneous oscillations in cerebral blood flow velocity in normal humans and in patients with carotid artery disease Neurosci. Lett. 127 5-8

Diehl R R, Linden D, Lucke D and Berlit P 1995 Phase relationship between cerebral blood flow velocity and blood pressure. A clinical test of autoregulation [see comments] Stroke 26 1801-4

Diehl R R, Linden D, Lucke D and Berlit P 1998 Spontaneous blood pressure oscillations and cerebral autoregulation Clin. Auton. Res. 8 7-12

Dimier-David L, Billon N, Costagliola D, Jaillon P and Funck-Brentano C 1994 Reproducibility of non-invasive measurement and of short-term variability of blood pressure and heart rate in healthy volunteers $\mathrm{Br}$. J. Clin. Pharmacol. 38 109-15

Giller C A 1990 The frequency-dependent behavior of cerebral autoregulation Neurosurgery 27 362-8

Hamon B V and Hannon E J 1974 Spectral estimation of time delay for dispersive and non-dispersive systems Appl. Stat. 23 134-42

Hetzel A, Eckenweber B, Trummer B, Wernz M, Schumacher M and von Reutern G 1998 Colour-coded duplex sonography of preocclusive carotid stenoses Eur. J. Ultrasound 38 183-91

Hu H H et al 1999 Transfer function analysis of cerebral haemodynamics in patients with carotid stenosis J. Cereb. Blood Flow Metab. 19 460-5

Kirkham S K, Craine R E and Birch A A 2001 A new mathematical model of dynamic cerebral autoregulation based on a flow dependent feedback mechanism Physiol. Meas. 22 461-73

Lang E W, Diehl R R and Mehdorn H M 2001 Cerebral autoregulation testing after aneurysmal subarachnoid haemorrhage: the phase relationship between arterial blood pressure and cerebral blood flow velocity Crit. Care Med. 29 158-63

Lassen N 1959 Cerebral blood flow and oxygen consumption in man Physiol. Rev. 39 183-238

Myers C W, Cohen M A, Eckberg D L and Taylor J A 2001 A model for the genesis of arterial pressure Mayer waves from heart rate and sympathetic activity Auton. Neurosci. 91 62-75

Panerai R B 1998 Assessment of cerebral pressure autoregulation in humans-a review of measurement methods Physiol. Meas. 19 305-38

Panerai R B, Deverson S T, Mahony P, Hayes P and Evans D H 1999 Effects of CO 2 on dynamic cerebral autoregulation measurement Physiol. Meas. 20 265-75

Panerai R B, Rennie J M, Kelsall A W and Evans D H 1998a Frequency-domain analysis of cerebral autoregulation from spontaneous fluctuations in arterial blood pressure Med. Biol. Eng. Comput. 36 315-22

Panerai R B, White R P, Markus H S and Evans D H 1998b Grading of cerebral dynamic autoregulation from spontaneous fluctuations in arterial blood pressure Stroke 29 2341-6

Preiss G and Polosa C 1974 Patterns of sympathetic neuron activity associated with Mayer waves Am. J. Physiol. 226 724-30

Reinhard M, Hetzel A, Lauk M and Lucking C H 2001 Dynamic cerebral autoregulation testing as a diagnostic tool in patients with carotid artery stenosis Neurol. Res. 23 55-63 
Timmer J, Lauk M, Häussler S, Radt V, Köster B, Hellwig B, Guschlbauer B, Lücking C H, Eichler M and Deuschl G 2000 Cross-spectral analysis of tremor time series Int. J. Bif. Chaos $102595-610$

von Reutern G and von Büdingen H J 1993 Ultrasound Diagnosis of Cerebrovascular Disease (Stuttgart: Thieme)

Widder B, Kleiser B and Krapf H 1994 Course of cerebrovascular reactivity in patients with carotid artery occlusions Stroke 25 1963-7

Young W L, Prohovnik I, Ornstein E, Ostapkovich N and Matteo R S 1991 Cerebral blood flow reactivity to changes in carbon dioxide calculated using end-tidal versus arterial tensions J. Cereb. Blood Flow Metab. 11 1031-5

Zhang R, Zuckerman J H, Giller C A and Levine B D 1998 Transfer function analysis of dynamic cerebral autoregulation in humans Am. J. Physiol. 274 H233-41 